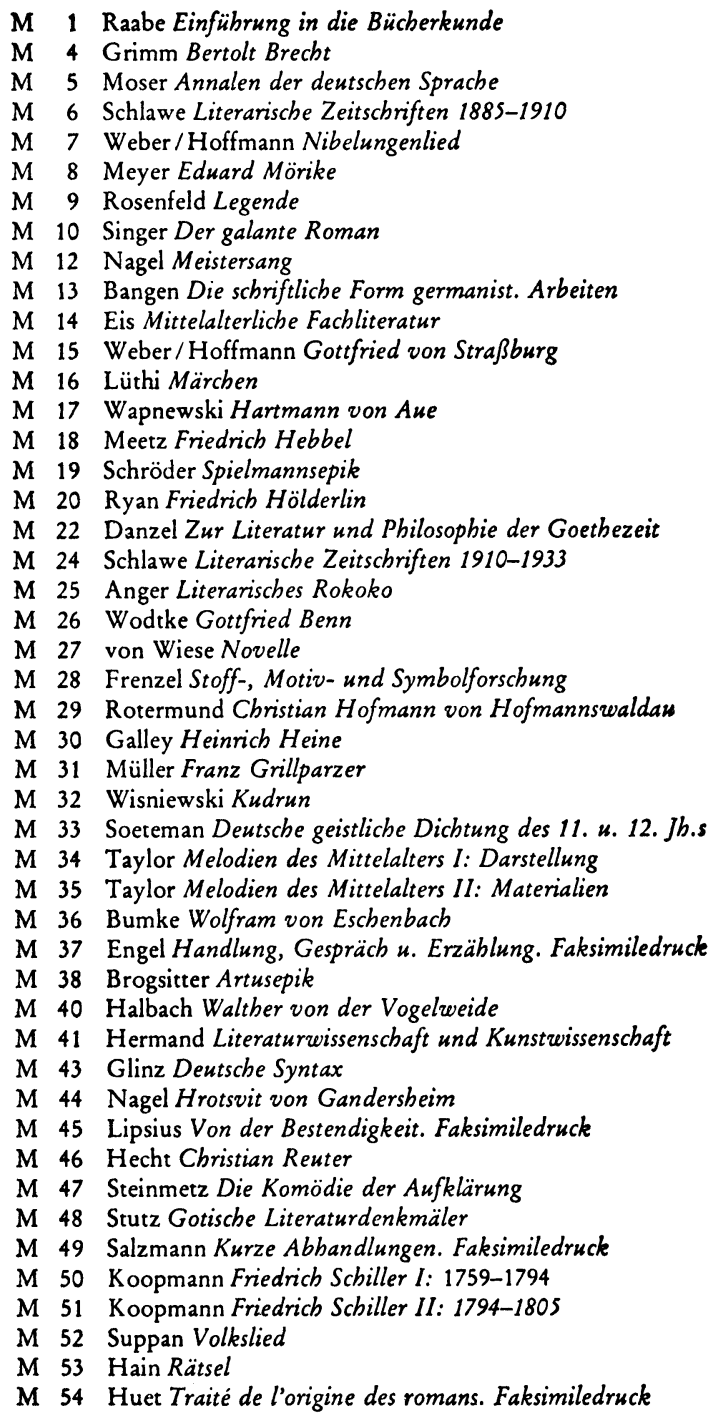




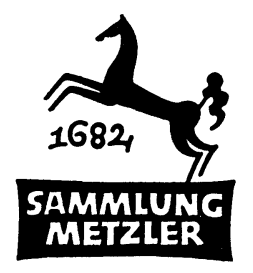

REALIEN ZUR LITERATUR

ABT. E:

POETIK 


\title{
Altdeutsche Metrik
}

\author{
2., überarbeitete
}

und ergänzte Auflage

MCMLXXXI

J. B. METZLERSCHE VERLAGSBUCHHANDLUNG

STUTTGART 
1. Aufl. (1. -5. Tsd.) 1967

2. Aufl. (6. -10 . Tsd.) 1981

CIP-Kurztitelaufnahme der Deutschen Bibliothek

Hoffmann, Werner:

Altdeutsche Metrik / Werner Hoffmann. 2., überarb. u. erg. Aufl. -

Stuttgart: Metzler, 1981.

(Sammlung Metzler; M 64: Abt. E, Poetik)

ISBN 978-3-476-12064-9

NE: GT

M 64

ISBN 978-3-476-12064-9

ISBN 978-3-476-04044-2 (eBook)

DOI 10.1007/978-3-476-04044-2

(C) Springer-Verlag GmbH Deutschland 1981

Ursprünglich erschienen bei J. B. Metzlersche Verlagsbuchhandlung und Carl Ernst Poeschel Verlag GmbH in Stuttgart 1981 


\section{VORWORT}

Das vorliegende Bändchen der 'Sammlung Metzler` soll den Leser mit den wichtigsten Fakten der altdeutschen Metrik vertraut machen. Dazu gehört nicht nur die Einführung in die Geschichte der altdeutschen Verskunst von ihren Anfängen bis zum Ausgang des Mittelalters, sondern auch - und hierauf habe ich besonderen Wert gelegt - die Einführung in die metrische Terminologie. Freilich: Die Fakten werden in der neuerdings wieder lebhaft in Fluß gekommenen Forschung vielfach nicht einheitlich gedeutet, die Grundbegriffe öfters abweichend definiert. Einführung in die "Realien « - die Grundlagen und den geschichtlichen Verlauf - der altdeutschen Verskunst kann darum immer nur bedeuten: Einführung in die derzeitige Forschungslage, ausdrücklicher Hinweis darauf, daß manches offen oder strittig ist. So muß der Leser, vielleicht $\mathrm{zu}$ seinem Mißbehagen, wiederholt mehrere, divergierende, Thesen kennenlernen. Andreas Heuslers als Ganzes nicht überholte, in vielen Einzelheiten allerdings der Revision bedürftige "Deutsche Versgeschichte « bildet dabei - auch hierin die Forschungslage widerspiegelnd - das Fundament meiner Darstellung, die daneben namentlich den Arbeiten Ulrich Pretzels verpflichtet ist.

Die Skizzierung gesicherter Fakten wie der Hinweis auf kontroverse Probleme ist nicht schon der eigentliche Sinn und Zweck eines Überblicks über die altdeutsche Metrik. Dieser ist vielmehr die Hinführung zum angemessenen Verständnis und zur Deutung dichterischer Texte. Die metrische Analyse und Interpretation ist Teil der Analyse und Interpretation des dichterischen Kunstwerks insgesamt. Die Metrik - als >Verskunde oder >Verslehre - muß hinführen zur >Verskunst $<$. Aber der Umgang mit Dichtung bedarf, wenn er nicht im Subjektiv-Unverbindlichen des gefühlhaften Eindrucks und Erlebens bleiben soll, eben des sachlich-begrifflichen Rüstzeuges wie der geschichtlichen Fundierung. Soweit es der begrenzte Raum erlaubt, soll diese Darstellung der altdeutschen Metrik hierfür die Grundlagen liefern. Daß dabei innerhalb des vorgegebenen Rahmens manches nicht oder nur kurz (und das heißt auch: unter Umständen vereinfachend) zur Sprache kommen kann, was an sich eine eingehendere und differenziertere Darlegung verdiente, versteht sich von selbst. Dies gilt namentlich auch für Grundbegriffe wie Akzent, Takt, Rhythmus, die im Grunde jeweils eine eigene Monographie erforderten.

Im Hinblick auf die sehr ausführliche Bibliographie in Ulrich Pretzels allgemein zugänglichem Beitrag über die »Deutsche Vers- 
kunst « in der 'Deutschen Philologie im Aufriß « wurden die Literaturangaben stark beschränkt und neben grundlegenden und zusammenfassenden älteren Werken vor allem (in Auswahl) repräsentative neuere Untersuchungen angeführt. Die Hinweise auf Pretzels Bibliographie beziehen sich immer auf die zweite Auflage der >Deutschen Philologie im Aufrißr. Im Text wird aus Pretzels "Deutscher Verskunst " grundsätzlich nur mit Angabe der Spaltenzahl nach der zweiten Auflage zitiert. Ebenso wird bei Andreas Heuslers häufig herangezogener "Deutscher Versgeschichte ", ohne ständige Wiederholung des Titels, nur der betreffende Paragraph genannt. Hervorhebungen innerhalb von Zitaten sind stets aus der Vorlage übernommen.

Frankfurt am Main, im Juli 1967

W.H.

\section{VORWORT ZUR 2. Auflage}

Seitdem die 1. Auflage meiner »Altdeutschen Metrik « erschienen ist, ist keine grundlegend neue Konzeption der deutschen Metrik vorgelegt worden. Die Zahl der Einzeluntersuchungen zur Metrik und zur deutschen Versgeschichte des Mittelalters hat sich dagegen beträchtlich vermehrt. Die neu hinzugekommenen Publikationen sind in der 2. Auflage in nicht zu knapper Auswahl verzeichnet und ihre Ergebnisse in die Darstellung eingearbeitet worden, wo immer dies aus sachlichen Gründen notwendig oder doch zweckmäßig war. Auch darüber hinaus habe ich manches hinzugefügt. Insgesamt soll die größere Ausführlichkeit vor allem einer stärkeren Präzisierung und Differenzierung der Aussagen, so in der Kennzeichnung des gegenwärtigen Forschungsstandes, dienen. Das mag gelegentlich zu Lasten seinfach zu lernender Sachverhalte gehen. Aber dieser mögliche Nachteil wird, wie ich meine, aufgewogen durch das Mehr an Informationen, die die 2. Auflage des Bändchens im Vergleich mit der 1. Auflage bietet.

Mannheim, im August 1980

W.H. 
1. Kapitel: Grundlagen und Grundbegriffe . . . . . . 1

2. Kapitel: Die altdeutsche Stabreimdichtung . . . . . 22

3. Kapitel: Otfrid von Weißenburg und der althochdeutsche Reimvers . . . . . . . . . . . 31

4. Kapitel: Die frühmittelhochdeutsche Zeit . . . . . 51

5. Kapitel: Der mittelhochdeutsche Reimpaarvers . . . . 64

6. Kapitel: Mittelhochdeutsche epische Strophenformen . 81

7. Kapitel: Metrik der mittelhochdeutschen lyrischen Dichtung ............... 97

8. Kapitel: Der deutsche Vers im ausgehenden Mittelalter und in der frühen Neuzeit - ein Ausblick . . 118

Sachregister . . . . . . . . . . . . . . . . . . 129 


\section{ABKüRZUngSVERZEICHNIS}

Sprachen:

$\begin{array}{ll}\text { afrz. } & \text { altfranzösisch } \\ \text { ahd. } & \text { althochdeutsch } \\ \text { anord. } & \text { altnordisch } \\ \text { as. } & \text { altsächsisch } \\ \text { bair. } & \text { bairisch } \\ \text { dt. } & \text { deutsch } \\ \text { frmhd. } & \text { frühmittelhochdeutsch } \\ \text { frnhd. } & \text { frühneuhochdeutsch } \\ \text { frz. } & \text { französisch } \\ \text { germ. } & \text { germanisch } \\ \text { hd. } & \text { hochdeutsch } \\ \text { idg. } & \text { indogermanisch } \\ \text { lat. } & \text { lateinisch } \\ \text { mhd. } & \text { mittelhochdeutsch } \\ \text { nhd. } & \text { neuhochdeutsch } \\ \text { prov. } & \text { provenzalisch } \\ \text { srhfrk. } & \text { südrheinfränkisch }\end{array}$

Grammatische Termini:

$\begin{array}{ll}\text { Dat. } & \text { Dativ } \\ \text { fem. } & \text { feminin } \\ \text { Gen. } & \text { Genitiv } \\ \text { Inf. } & \text { Infinitiv } \\ \text { mask. } & \text { maskulin } \\ \text { Pass. } & \text { Passiv } \\ \text { Pl. } & \text { Plural } \\ \text { Sg. } & \text { Singular }\end{array}$

Dichtungstitel (nur in Verbindung mit Stellenangaben):

aH.

Hartmann von Aue: Der arme Heinrich

Gr.

$\mathrm{Hl}$.

Hartmann von Aue: Gregorius

Ludw. Hildebrandslied

MF

Des Minnesangs Frühling

NL Nibelungenlied

$\mathrm{O} \quad$ Otfrid von Weißenburg: Evangelienbuch

Parz. Wolfram von Eschenbach: Parzival

Tr. Gotfrid von Straßburg: Tristan (Verszählung nach der Ausgabe Friedrich Rankes)

Wh. Wolfram von Eschenbach: Willehalm

Sonstiges:
Diss.
Dissertation
Hs.
Handschrift 
$\begin{array}{ll}\text { Str. } & \text { Strophe } \\ \text { v. } & \text { Vers }\end{array}$

Wissenschaftliche Reiben, Zeitschriften, Sammelwerke:

ABÄG

AfdA

Arch.

ATB

Aufriß

Beitr.

DU

DVjs.

Et. Germ.

Euph.

GAG

GGA

GRM

HSB

JbIG

LJb.

MGG

MLR

MTU

Neuphil. Mitt. Neuphilologische Mitteilungen

RL

SM

WdF, Bd. 15 Der deutsche Minnesang. Aufsätze zu seiner Erforschung, hg. von Hans Fromm, 1961, ${ }^{5} 1972$ (= Wege der Forschung, Bd. 15)

WdF, Bd. 154 Mittelhochdeutsche Spruchdichtung, hg. von Hugo Moser, 1972 (= Wege der Forschung, Bd. 154)

WdF, Bd. 444 Die Genese der europäischen Endreimdichtung, hg. von Ulrich Ernst und Peter-Erich Neuser, 1977 ( = Wege der Forschung, Bd. 444)

WSB

Sitzungsberichte der Akademie der Wissenschaften zu Wien. Philologisch-historische Klasse

WW Wirkendes Wort

ZfdA Zeitschrift für deutsches Altertum und deutsche Literatur

ZfdPh Zeitschrift für deutsche Philologie 